\title{
CONCAVE SOLUTIONS OF A DIRICHLET PROBLEM
}

\section{H. GUGGENHEIMER ${ }^{1}$}

\begin{abstract}
We define a function that may serve as the measure of the deviation of a plane convex domain from circular shape. Then we show that the Dirichlet problem of $\Delta u+\lambda p(x, y) u=0$ and vanishing boundary values on a plane, convex domain can have a concave solution only if an integral condition involving the deviation from circular shape is satisfied. A weak form of the condition is generalized to $n$ dimensions.
\end{abstract}

The basic estimate for the first eigenvalue of a problem

$$
\begin{aligned}
& x^{\prime \prime}+\lambda p(t) x=0, \quad p(t) \geqq 0, \\
& x(a)=x(b)=0,
\end{aligned}
$$

is Ljapunov's inequality

$$
\lambda_{1} \int_{a}^{b} p(t) d t \geqq 4 /(b-a) .
$$

The estimate has been generalized to $p(t)$ of varying sign and to partial differential equations.

Joukovsky [4] has given an elegant proof of (1) based on the fact that the graph of the first eigenfunction together with the segment $[a, b]$ bounds a convex domain. In this note, we use a method inspired by that of Joukovsky to study concave solutions of

$$
\Delta u+\lambda p(x) u=0, \quad p(x) \geqq 0,
$$

defined in a convex domain $D$ of real $n$-space of coordinates $x=$ $\left(x_{1}, \cdots, x_{n}\right)$ for vanishing boundary values, $\left.u\right|_{\partial D}=0$.

For given $p$, the behavior of the solution obviously depends on the geometry of $D$. The important features can be studied on the example of the equation with constant coefficients,

$$
\Delta u+\lambda u=0 \text { in } D, \quad u=0 \text { on } \partial D .
$$

Received by the editors September 15, 1972 and, in revised form, December 18, 1972. AMS (MOS) subject classifications (1970). Primary 35J25, 52A10; Secondary 35P15, $52 \mathrm{~A} 20$.

${ }^{1}$ Research partially supported by NSF Grant GP-27960.

(c) American Mathematical Society 1973 
For $n=2$ and $D$ the unit disc, the solution surface

$$
u_{1}=J_{0}\left(j_{0}\left(x_{1}^{2}+x_{2}^{2}\right)^{1 / 2}\right)
$$

is concave and together with $D$ bounds a convex body $\left(j_{0} \cong 2.4048\right.$ is the first zero of the Bessel function of order zero). In this case,

$$
\lambda_{1} \int_{D} p(x) d x_{1} d x_{2}=j_{0}^{2} \pi \text {. }
$$

For $D$ the square $[0, \pi] \times[0, \pi]$, the solution surface $u_{1}=\sin x_{1} \sin x_{2}$ is not concave and $\lambda_{1} \int_{D} p(x) d x_{1} d x_{2}=2 \pi^{2}$.

The inequality we shall prove indicates that the energy represented by $\lambda_{1} \int_{D} p d x_{1} d x_{2}$ must be large if $u_{1}$ is to be concave for noncircular $D$. For the square, the inequality would just replace $=$ by $>$. The inequality is rather strong for dimension 2 but weak for dimensions $>2$.

Deviation from circular shape. Let $c$ be a strictly convex, closed, plane curve and $h(\varphi)$ its support function with respect to an interior point 0 . We put

$$
s(\varphi)=(\cos \varphi, \sin \varphi), \quad n(\varphi)=(-\sin \varphi, \cos \varphi) .
$$

The point of $c$ whose oriented line of support has direction $n(\varphi)$ is

$$
c(\varphi)=h(\varphi) s(\varphi)+h^{\prime}(\varphi) n(\varphi) .
$$

We denote by $\mu$ the angle between $c(\varphi)$ and $n(\varphi)$ :

$$
\cot \mu(\varphi)=h^{\prime}(\varphi) / h(\varphi) \text {. }
$$

For a circle referred to its origin, $\cot \mu \equiv 0$. Conversely, a curve for which $\cot \mu$ vanishes identically is a circle of origin 0 .

In imitation of the definition of measures of symmetry [3] we define a function on the space of plane, proper, convex bodies to be a measure of deviation from circular shape if

(i) the function is invariant under the group of similarities,

(ii) the function is nonnegative and vanishes only for the circle.

The angle $\mu(\varphi)$ can serve to define such a measure.

Proposition 1. $H=\min _{0 \in \mathrm{int}} \oint \oint \cot ^{2} \mu(\varphi) d \varphi$ is a measure of deviation from circular shape.

The minimum exists since the integral is nonnegative, tends to $\infty$ for 0 tending to a point of the boundary $c$ and, as will follow from our computations, the integrand is uniformly continuous if 0 is in the interior of $c$. Condition (i) follows from the invariance of angles and (ii) from the remark after the definition (2).

Proposition 2. In the interior of any closed, convex curve there is a unique point at which $\oint \cot ^{2} \mu(\varphi) d \varphi$ is minimal. 
We assume first that $c$ is a strictly convex curve. If we move 0 to $0^{*}$ by a translation of vector $\varepsilon$, the support function is changed from $h(\varphi)$ to $h^{*}(\varphi)=h(\varphi)-\varepsilon \cdot s(\varphi)$. Hence, the integrand is changed to

$$
\begin{aligned}
\cot ^{2} \mu^{*}(\varphi)= & h^{* \prime 2} / h^{* 2} \\
= & \frac{h^{\prime 2}}{h^{2}}\left\{1+2\left[\frac{\varepsilon \cdot s}{h}-\frac{\varepsilon \cdot n}{h^{\prime}}\right]+\frac{(\varepsilon \cdot n)^{2}}{h^{\prime 2}}\right. \\
& \left.+3 \frac{(\varepsilon \cdot s)^{2}}{h^{2}}-4 \frac{(\varepsilon \cdot s)(\varepsilon \cdot n)}{h h^{\prime}}+o\left(|\varepsilon|^{2}\right)\right\} .
\end{aligned}
$$

Since $\varepsilon$ is an arbitrary vector, the integrand is stationary as a function of 0 only if

$$
\oint \frac{h^{\prime 2}}{h^{2}}\left(\frac{s}{h}-\frac{n}{h^{\prime}}\right) d \varphi=0 .
$$

We are interested in the quadratic term

$$
\frac{(\varepsilon \cdot n)^{2}}{h^{\prime 2}}+3 \frac{(\varepsilon \cdot s)^{2}}{h^{2}}-4 \frac{(\varepsilon \cdot s)(\varepsilon \cdot n)}{h h^{\prime}} \geqq 2 \frac{(\varepsilon \cdot s)^{2}}{h^{2}} .
$$

Since the term is nonnegative and its integral positive, any stationary point is an isolated minimum. Since the Euler characteristic of a convex domain is one and the stationary points are nondegenerate, Morse theory (in this simple case, a formula of C. Maxwell) implies that there is a unique stationary point.

For a curve that is not strictly convex, $\mu(\varphi)$ is not defined if the line of direction $n(\varphi)$ carries a segment of $c$. However, $\mu(\varphi)$ is defined by (2) for all $\varphi$ except a countable set and the conclusion of the preceding paragraph still holds.

EXAmple. For the square, 0 has to be chosen as the center and $H=$ $8-2 \pi$.

\section{A condition for concave solutions.}

Proposition 3. If the first eigenfunction of $\Delta u+\lambda p(x) u=0, p(x)>0$, in the interior of a closed, convex curve $c, u=0$ on $c$, is concave then

$$
\lambda_{1} \int_{\text {int } c} p(x) d x_{1} d x_{2}>2 \pi+H(c)
$$

where $H(c)$ is the deviation of the curve $c$ from circular shape.

Proof. We may take the eigenfunction $u_{1}>0$ in int $c$. By definition,

$$
\int_{\text {int } c} \Delta u_{1} d x_{1} d x_{2}=-\lambda_{1} \int_{\text {int } c} p(x) d x_{1} d x_{2}
$$


and, by the Green-Stokes formula,

$$
\int_{\text {int } c} \Delta u_{1} d x_{1} d x_{2}=-\oint_{c}\left|\operatorname{grad} u_{1}\right| d s
$$

since $c$ is a level line of the function $u_{1}$. Here, $d s$ is the element of length of the convex curve $c$.

The convexity of the surface $u_{1}=u_{1}\left(x_{1}, x_{2}\right)$ implies first that $u_{1}$ has a unique maximum $m=u_{1}(P)$ in int $c$. Let $h$ be the support function for the center $P$. Since the tangent plane at any point $Q \in c$ is in the exterior of the surface, $m \leqq h(Q)\left|\operatorname{grad} u_{1}(Q)\right|$. Hence,

$$
\begin{aligned}
m \lambda_{1} \int_{\text {int } c} p d x_{1} d x_{2} & \geqq \lambda_{1} \int_{\text {int } c} p u_{1} d x_{1} d x_{2} \\
& =\oint_{c}\left|\operatorname{grad} u_{1}(Q)\right| d s(Q) \geqq m \oint_{c} \frac{d s(Q)}{h(Q)} .
\end{aligned}
$$

The last integral appears in classical eigenvalue estimates (cf. [2, §11.3]).

For the moment, we assume that $c$ is a $C^{2}$ curve. Then, $d s=\left(h+h^{\prime \prime}\right) d \varphi$ and

$$
\oint \frac{d s}{h}=\int_{0}^{2 \pi}\left(1+\frac{h^{\prime \prime}}{h}\right) d \varphi=2 \pi+\int_{0}^{2 \pi}\left(\frac{h^{\prime}}{h}\right)^{2} d \varphi \geqq 2 \pi+H .
$$

Since any closed, convex curve can be approximated in the Blaschke topology by analytic curves (see, e.g., [1, no. 27]) and the terms at the extremes of (4) are defined for all convex curves and lower semicontinuous in the Blaschke topology, the inequality (4) holds for all closed, convex curves in the form $\oint h^{-1} d s \geqq 2 \pi+H$. We also note that equality cannot hold between the extreme terms of (3) even if we would allow $p(x)$ to be a generalized function. In fact, equality in the first inequality can hold only if the surface $u=u_{1}(x)$ is a right cylinder over $c$ and in the second inequality if it is a cone. The proposition now follows from (3) and (4).

The strict inequality in the proposition is essential as can be seen from the example of the vibrating plate $(p=1)$ for which nevertheless

$$
2 \pi^{2}=\lambda_{1} \int_{\text {int } c} p d x_{1} d x_{2}>\lambda_{1} \int_{\text {int } c} p u_{1} d x_{1} d x_{2}=8=2 \pi+H .
$$

By the preceding remark, the fact that $u_{1}$ cannot be concave is contained in the last equality. Therefore, we may state:

COROLlaRY. The first eigenfunction $u_{1}$ cannot be concave if

$$
\lambda_{1} \int_{\text {int } c} p(x) u_{1}(x) d x_{1} d x_{2}=2 \pi+H \text {. }
$$


An inequality for higher dimensions. Let $P$ be an interior point of a convex domain $D$ in $n$-space. The polar equation of the boundary of $D$ for the origin at $P$ is $r=r(s)$ where $s \in S^{n-1}$ is the unit vector on the ray from $P$ to the point on $\partial D$. The line of direction $s$ intersects $D$ in a chord of length $\delta(s)=r(s)+r(-s)$. The $(n-1)$-volume element of the unit sphere $S^{n-1}$ is $d \omega$; we denote the surface area of the $(n-1)$ unit sphere by $c_{n-1}$ and put $c_{0}=2$. We also write $d V$ for the volume element in $n$-space, $d S$ for the $(n-1)$ volume element on the boundary surface $\partial D$, and put

Then,

$$
\delta(P)=c_{n-1}^{-1} \int_{S^{n-1}} \delta(s) d \omega(s) .
$$

$$
\delta^{*}(D)=\min _{P \in D} \delta(P) \geqq w(D)
$$

where $w(D)$ is the width, the minimal distance of parallel support planes. With these notations, we have:

PRoposition 4. If the problem $\Delta u+\lambda p(x) u=0$ in a convex domain $D$ in $n$-space, $p(x)$ nonnegative and integrable in $D, u=0$ on $\partial D$, admits a first eigenfunction $u_{1}$ defining a convex hypersurface $x_{n+1}=u_{1}\left(x_{1}, \cdots, x_{n}\right)$ in $(n+1)$-space then

$$
\lambda_{1} \int_{D} p(x) d V \geqq c_{n-1}\left(\frac{\delta^{*}(D)}{2}\right)^{n-2} .
$$

Equality cannot hold for $n>1$.

For $n=1$, equality holds for $p$ a certain $\delta$-function since, then, the proposition reduces to Ljapunov's inequality (1). For $n>1$, equality is excluded for the same reason as in Proposition 3. The proof of Proposition 4 is an imitation of that of Proposition 3:

$$
\begin{aligned}
m \lambda_{1} \int_{D} p(x) d V & \geqq \lambda_{1} \int_{D} p(x) u_{1}(x) d V=\int_{\partial D}\left|\operatorname{grad} u_{1}(Q)\right| d S \\
& \geqq m \int_{\partial D} \frac{d S}{h(Q)} .
\end{aligned}
$$

Put $r=|Q-P|$ and let $s \in S^{n-1}$ be the unit vector in direction $Q-P$. Then,

$$
\int_{\partial D} \frac{d S}{h(Q)} \geqq \int_{\partial D} \frac{d S}{r} \geqq \int_{S^{n-1}} r^{n-2} d \omega(s) .
$$

We may suppose $n>2$ since, for $n=2$ Proposition 3 is stronger than Proposition 4. By the Hölder inequality,

$$
c_{n-1}^{n-3} \int_{S^{n-1}} r^{n-2} d \omega \geqq\left(\int_{S^{n-1}} r d \omega\right)^{n-2},
$$


or,

$$
\begin{aligned}
\int_{S^{n-1}} r^{n-2} d \omega & \geqq c_{n-1}\left\{c_{n-1}^{-1} \int_{S^{n-1}} r d \omega\right\}^{n-2} \\
& =c_{n-1}(\delta(P) / 2)^{n-2} .
\end{aligned}
$$

The proposition follows at once.

\section{REFERENCES}

1. T. Bonnesen und W. Fenchel, Theorie der konvexen Körper, Springer, Berlin, 1934.

2. P. R. Garabedian, Partial differential equations, Wiley, New York, 1964. MR 28 \#5247.

3. Branko Grünbaum, Measures of symmetry for convex sets, Proc. Sympos. Pure Math., vol. 7, Amer. Math. Soc., Providence, R.I., 1963, pp. 233-270. MR 27 \#6187.

4. N. E. Joukovsky, Conditions of boundedness of integrals of equations $\left(d^{2} y / d x^{2}\right)+$ $p y=0$, Mat. Sb. 16 (1891/93), 582-591. (Russian)

Department of Mathematics, Polytechnic Institute of Brooklyn, Brooklyn, NEW YORK 11201 